\section{Rotation groups about a set of fixed points.}

By

Lucille Whyburn (Virginia, U. S. A.).

This paper concerns itself with the set of points that remain fixed under a continuous one to one transformation of a set $M$ in a topological space into itself. We shall show that the components of the complement of such a set of fixed points in $M$ fall into groups of two types, one composed of a finite number of elements and the other of an infinite number. By putting restrictions on $M$ or on the group of components or both we are able to establish certain properties of the components and of our set of fixed points: for example, if $M$ is a plane continuous curve, every component of $M$ minus the set of fixed points under any homeomorphism $T(M)=M$. has property $S$, or if $M$ is a sphere and there exists one group of components (in the above sense) containing at least two elements, then our set of fixed points is a simple closed curve and our transformation $T$ must be such that it merely interchanges the two complementary regions of this simple closed curve.

\section{Preliminary Notions and Theorems.}

Definitions. A collection $C_{0}, C_{1}, C_{2}, \ldots, C_{n}, \ldots$ of components ${ }^{1}$ ) of $M-K$, where $M$ is a point set and $K$ is the set of fixed points under a homeomorphism $T(M)=M$, is said to form a rotation group of Type I (finite rotation group) provided (1) the collection $C_{0}, C_{1}, C_{2}, \ldots, C_{i}, \ldots$ contains only a finite number of components, say $n+1$, and (2) the components of the collection may be ordered in such a way that $T\left(C_{0}\right)=C_{1}, T\left(C_{1}\right)=C_{2}, \ldots T\left(C_{t}\right)=C_{i+1} \ldots T\left(O_{n}\right)=C_{0}$

1) A component of a point set $S$ is a maximal connecter subset of $S$. and so that $T\left(C_{j}\right) \neq C_{i}$ for $i<j<n$; such a collection is said to form a rotation group of Type II (infinite rotation group) provided

(1) the collection contains infinitely many components,

(2) it may be ordered as follows,

$$
\ldots, C_{-n}, \ldots, C_{-1}, C_{0}, C_{1}, C_{2}, \ldots C_{n}^{\prime}, \ldots,
$$

where $T\left(C_{i}\right)=C_{i+1}$ and $T\left(C_{j}\right) \neq C_{i}$ for $i<j$.

It is at once apparent that for any two elements $C_{i}$ and $C_{j}$ of either type of rotation group $C_{i}=T^{i-j}\left(C_{j}\right)$ and $C_{j}=T^{j-i}\left(C_{i}\right)$.

Moreover both the infinite and finite rotation groups form cyclic groups under the following definition of the group operation: $C_{i} C_{j}=T^{\prime}\left(C_{i}\right)$.

Hereafter $K$ will always denote the set of fixed points under a homeomorphism $T(M)=M$. Clearly $K$ is always a closed set.

Theorem 1. If $T(M)=M$ is a homeomorphism and $K$ is the set of fixed points under $T$, then every component of $M-K$ falls into one and only one rotation group.

Proof. Take any component $c$ of $M-K$ and call it $C_{0}$. Now since $K$ is a set of fixed points under $T$ and $T$ is one to one, $T(M-K)=M-K$. Now we wish to show that $T\left(C_{0}\right)$ is a component of $T(M-K)$ and consequently of $M-K$. In the first place since $C_{0}$ is connected and $T$ is continuous, $T\left(C_{0}\right)$ is connected and is consequently contained in some component $Q$ of $T(M-K)$; secondly, $T$ is a homeomorphism and therefore $T^{-1}$ is one to one and continuous and consequently $T^{-1}(Q)$ is connected and contains $C_{0}$, from which, since $C_{0}$ is a component of $M-K$, it follows that $T^{-1}(Q) \equiv C_{0}$; and from this fact it follows immediately that $T\left(C_{0}\right) \equiv Q$, i. e., $Q=T\left[T^{-1}(Q)\right]=T\left(C_{0}\right)$. Thus $T\left(C_{0}\right)$ is a component of $M-K$.

Now let us designate $T\left(C_{0}\right)$ as $C_{1}$. Then, just as above, $T\left(C_{1}\right)$ is a component of $M-K$, and we set $C_{2}=T\left(C_{1}\right) ; \quad T\left(C_{2}\right)$ is a component of $M-K$, and we set $C_{3}=T\left(C_{2}\right)$, etc. ... If for any $n=1,2,3, \ldots, T\left(C_{n}\right)=C_{0}$, we shall consider our selection at an end. And we see at once that we have a group of Type I provided we can show that for no integer $k$ can $T\left(C_{k}\right)=C_{i} \quad(i<k<n)$. To see this let $m$ be the smallest integer $k$ such that $T\left(C_{k}\right)=C_{i}(i<k<n)$. 
Then since $m<n, i \neq 0$, which gives us $T\left(C_{i-1}\right)=C_{i}$ and $T\left(C_{m}\right)=C_{l}$, contrary to the fact that $T$ is one to one because $C_{l-1} \neq C_{m}$ or $(m-1)$ would have been the first $k$ such that $T\left(C_{k}\right)=C_{l} \quad(i<k<n)$. If, then, there exists an $n$ such that $T\left(C_{n}\right)=C_{0}$, we have a group of Type $\mathrm{I}$.

If, however, $T\left(C_{n}\right) \neq C_{0}$ for every positive value of $n$, it is easy' to see we get an infinite sequence $C_{0}, C_{1}, C_{2}, \ldots, C_{n}, \ldots$ such that $T\left(C_{i}\right)=C_{i+1}$. Furthermore, since $T$ is a homeomorphism and $C_{0}$ is a component of $M-K, T^{-1}\left(C_{0}\right)$ must be a component of $M-K$. Let us set $T^{-1}\left(C_{0}\right)=C_{-1}$, and $T^{-1}\left(C_{-1}\right)=C_{-2}$, etc. An argument almost identical with the one given above to show that $T\left(C_{k}\right) \neq C_{i}(i<k<n)$ may be used to show that this negative series can not terminate or coincide with the positive series in any component; and a similar argument may be used to show that the series composed of the sum of the negative and positive series satisfies the condition that $T\left(C_{j}\right) \neq C_{i}$ for $i<j$. Thus we have a collection

$$
\ldots, O_{-n}, \ldots, C_{-3}, C_{-2}, C_{-1}, C_{0}, C_{1}, C_{2}, \ldots, C_{n}, \ldots
$$

satisfying the definition of a rotation group of Type II.

Starting with any component $c$ of $M-K$ we have shown that we can pick out a rotation group of Type I or Type II containing that element.

It remains only to show that $c$ cannot belong to more than one rotation group. This follows very simply from the fact that if $a$ belongs to a given rotation group so also does $T(c)$.

Theorem 2. If $M$ is a set of points and $T(M)=M$ is a homeomorphism then for any two elements $C_{i}$ and $C_{j}$ of a rotation group, $\bar{C}_{i}-C_{i}=\bar{C}_{j}-C_{j}$.

Proof. If we denote the set $\bar{C}_{i}-C_{i}$ by $F\left(C_{i}\right)$, then $F\left(C_{i}\right) \subset K$. Therefore if $P \in F\left(C_{i}\right), T(P)=P$. Now let us choose any point $P \in F\left(C_{i}\right)$ and a set of points $p_{1}, p_{2}, p_{3}, \ldots$ of $C_{i}$ converging to $P$ and proceed to show $P \in F\left(C_{j}\right)$. As noted in the introduction, $C_{j}=T^{j-i}\left(C_{i}\right)$, and since this transformation is continuous, limit points are preserved; therefore $T^{j-i}\left(p_{1}\right), T^{j-i}\left(p_{2}\right), \ldots$ must have as a limit point $T^{j-i}(P)$; but $T^{j-i}(P)=P$, since $P \in K$. Thus we have shown that if $P_{\epsilon} F^{\prime}\left(C_{i}\right), P_{\epsilon} F^{\prime}\left(C_{j}\right)$. Hence also $P \in F\left(O_{j}\right)$ implies $P \in F^{\prime}\left(C_{i}\right)$. Thus $F\left(C_{i}\right)=F^{\prime}\left(C_{j}\right)$ and our theorem is proven.
Corollary. If $M$ is connected and locally connected it follows that for any rotation group, $F\left(\sum_{i} C_{i}\right)=\sum_{i} F\left(C_{i}\right)=F\left(C_{i}\right)$.

Corollary. Under the conditions of Theorem 2, if $C$ is an element of a rotation group under $T$, any point $P \in F(C)$ which is accessible ${ }^{1}$ ) from $C$ is accessible from any element of $C$ 's.rotation group.

\section{Rotation Groups in a Plane Continuous Curve.}

Theorem 3. If $M$ is a plane continuous curve and $T(M)=M$ is a homeomorphism and $C$ is an element of a rotation group under $T$ of order $\geqslant 2$, then $C$ has property $S^{2}{ }^{2}$.

Proof. Let us suppose that $C$ does not have property $S$. Then there exists an $\varepsilon>0$ and a sequence of points $p_{1}, p_{2}, p_{3}, \ldots$ of $C$ converging to a point $P$ of $F(C)$ such that no two of these points can be joined in $C$ by a connected set of diameter $<\varepsilon$. About the point $P$ take a circle $N$ of diameter $\varepsilon / 2$. Clearly we may suppose all the points $p_{1}, p_{2}, p_{3}, \ldots$ lie within $N$. Then for each $j$ there exists an arc $p_{j} q_{j}$ in $C$ such that $p_{j} q_{j} \cdot N=q_{j}$. Manifestly the arcs $p_{j} q_{j}$ are disjoint and we may suppose they converge to a limit continuum $H$ which lies in $\bar{C}$. Now, if we show that $H \cdot C=0$, it follows that, $H \subset F(C) \subset K$. Suppose, then, that $b \in H \cdot O$. Since $C$ is locally connected, there exists a $\delta$ such that any point of $C$ at a distance $<\delta$ from $b$ may be joined in $C$ to $b$ by an are lying within $N$. Since $b \in H$, infinitely many of the $\operatorname{arcs} p_{j} q_{j}$ contain points at a distance $<\delta$ from $b$. Let $p_{s} q_{s}$ and $p_{t} q_{t}$ be two such arcs. Then $b$ can be joined to $p_{s} q_{s}$ and also to $p_{t} q_{t}$ by an arc of $C$ lying within $N$, and it is easy to see that these four arcs form a connected set in $C$ of diameter $<\varepsilon$ containing $p_{s}$ and $p_{t}$ contrary to our assumption that no two of the points $p_{1}, p_{2}, p_{3}, \ldots$ can be joined in $C$ by a connected set of diameter $<\varepsilon$. Thus $H \cdot C=0$ and we have $H \subset F(C) \subset K$. We see then that the disjoint $\operatorname{arcs} p_{j} q_{j}$ converge to a limit continuum $H$

1) A point $p$ is said to be accessible from a set of points $R$ provided there exists a simple arc $x p$ contained in $R+p$.

2) A set of points $Q$ is said to have property $S$ provided that for any preas: signed positive number $\varepsilon, Q$ is the sum of a finite number of connected sets of diameter less than $\varepsilon$. See W. Sierpiński, Sur une condition pour qu'un continu soit une courbe jordanienme, Fund. Math. 1 (1920), pp. 44-60. See also R. L. Moore, soit une courbe jordanienne, Fund. Math. 1 (1920), pp.44-60. See also R. L. Moore, pp. 232-237. 
such that $P \subset H \subset F(C) \subset K$. Let $P^{\prime} \in H$ and $P^{\prime} \neq P$. And since $H$ contains both $P$ and a point of $N$ we may suppose $P^{\prime}$ chosen so that $\varrho\left(P^{\prime}, P\right)=\varepsilon / 4$. Let $N^{\prime}$ be a circle with center $P^{\prime}$ and of diameter less than $\varepsilon / 4$ which encloses no point of the sequence $p_{1}, p_{2}, p_{3}, \ldots$ Then it follows that there exists a sequence of points $p_{1}^{\prime}, p_{0}^{\prime}, p_{3}^{\prime}, \ldots$ converging to $P^{\prime}$ and such that for each $p_{j}^{\prime}$ there exists an arc $r_{j} p_{j}^{\prime} s_{j} \subset p_{j} q_{j}$ such that $r_{j} p_{j}^{\prime} s_{j} \cdot N^{\prime}=r_{j}+s_{j}$. There exists an integer $G$ such that if $m$ and $n$ are greater than $G$ then $p_{m}^{\prime}$ and $p_{n}^{\prime}$ lie together in an are of $M$ lying wholly within $N^{\prime}$. Take any three distinct indices $n, m, k>G$. Of the three arcs $r_{n} p_{n}^{\prime} s_{n}, r_{m l} p_{m}^{\prime} s_{m}, r_{k} p_{k}^{\prime} s_{k}$, one, say $r_{m} p_{m}^{\prime} s_{m}$, separates the other two in the circle $N^{\prime}$ plus its interior. Let us take the $\operatorname{arcs} p_{m}^{\prime} p_{n}^{\prime}$ and $p_{m}^{\prime} p_{k}^{\prime}$ lying within $N^{\prime}$ and in $M$, as above. These ares contain sub-arcs $u_{m} v_{n}$ and $z_{m} v_{k}$ such that $u_{m} v_{n} \cdot r_{n} p_{n}^{\prime} s_{n}=v_{n}, u_{m} v_{n} \cdot r_{m} p_{m}^{\prime} s_{m}=u_{n}, z_{m} w_{k} \cdot r_{m} p_{u_{k}}^{\prime} s_{n}=z_{m}$, and $z_{m l} w_{k} \cdot v_{k} p_{k}^{\prime} s_{k}=w_{k}$. Since $\delta\left(p_{m} q_{m}+u_{m} v_{n}+p_{n} q_{n}\right)<\varepsilon$, it follows that $u_{m} v_{n} \cdot K \neq 0$ and similarly $z_{m} w_{k} \cdot K \neq 0$. Let $x_{1}$ be the first point of $u_{m} v_{n}$ in the order from $u_{m}$ to $v_{n}$ belonging to $K$, and likewise $y_{1}$ will designate the first point of $z_{m} w_{k}$ in the order from $z_{m}$ to $w_{k}$ of $K$. Then $x_{1} y_{1}-\left(x_{1}+y_{1}\right)$ is contained in $C$ and $x_{1}$ is separated from $y_{1}$ in $N^{\prime}$ plus its interior by the arc $r_{m} p_{m}^{\prime} s_{m}$. But $T\left(x_{1} y_{1}\right)$ must contain a point $b_{1}$ lying outside $N^{\prime}$ because since $x_{1}, y_{1} \in K, T\left(x_{1}\right)=x_{1}$ and $T\left(y_{1}\right)=y_{1}$, and therefore $T\left(x_{1}\right)$ is separated from $T\left(y_{1}\right)$ in $N^{\prime}$ plus its interior by $r_{m} p_{m}^{\prime} s_{m}$, it follows that $T\left(x_{1} y_{1}\right)-\left(x_{1}+y_{1}\right)$ must either contain a point lying outside of $N^{\prime}$ or contain a point of $r_{m} p_{m l}^{\prime} s_{m}$, whereas $r_{m} p_{m}^{\prime} s_{m} \subset C, T\left(x_{1} y_{1}\right)-\left(x_{1}+y_{1}\right) \subset T(C)$ and $T(C) \cdot C=0$ by definition. Taking any three distinct indices greater than $m, n$, or $k$ and following the same procedure as above we may obtain an arc $x_{2} y_{2}$ lying within $N^{\prime}$ plus its interior and such that $T\left(x_{2} y_{2}\right)$ contains a point $b_{2}$ lying outside $N^{\prime}$ plus its interior and satisfying the other conditions of the are $x_{1} y_{1}$. Repeating this process indefinitely we obtain an infinite sequence of $\operatorname{arcs} x_{1} y_{1}, x_{2} y_{2}, \ldots$, which may be chosen so as to converge to $P^{\prime}$ but such that $T\left(x_{i} y_{i}\right)$ contains a point $b_{i}$ lying outside $N^{\prime}$ plus its interior. Clearly this is impossible since $T^{-1}\left(b_{i}\right) \rightarrow P^{\prime}$, and $T\left(P^{\prime}\right)=P^{\prime}$ whereas $T T^{-1}\left(b_{i}\right)=b_{i}$ and the sequence $b_{i}$ does not converge to $T\left(P^{\prime}\right)$.

Corollary. Under the conditions of Theorem 3, every point of $F(C)$ is accessible from $C$.
Theorem 4. If $M$ is a plane continuous curve and $C$ is an. element of a rotation group of order $\geqslant 2$, then $F(C)$ is contained in some simple closed curve.

This theorem follows immediately from the above corollary and a theorem of $G$. T. Whyburn ${ }^{1}$ ).

Theorem 5. If $M$ is a plane continuous curve and $T(M)=M$ is a homeomorphism and $F(C)$ [C being a component of $M-K]$ contains more than two points, then the rotation group of $C$ is of order $\leqq 2$.

Proof. Suppose the theorem is not true. Then the rotation group of $C$ is at least of order three and we may assume that $T(C)=D \neq C, T^{2}(C)=T(D)=E \neq D$ and $E \neq C$. Now we proceed to show that this assumption leads to a contradiction. To do this let us choose any three points $x, y$ and $z$ of $F(C)$. By the Corollary following Theorem 3, taking some point $p_{\epsilon} O$ we can obtain three ares $p x, p y, p z$ in $C+x+y+z$; and as a subset of the sum of these three arcs we can pick out three arcs $p^{\prime} x, p^{\prime} y, p^{\prime} z$ each two of which have just $p^{\prime}$ in common. From this we have the arcs $T\left(p^{\prime} x\right)$, $T\left(p^{\prime} y\right)$, and $T\left(p^{\prime} z\right)$ lying in the set $D+x+y+z$, each two of which have just the point $T\left(p^{\prime}\right)$ in common. Likewise, we obtain the ares $T^{2}\left(p^{\prime} x\right), T^{2}\left(p^{\prime} y\right)$, and $T^{2}\left(p^{\prime} z\right)$ lying in $E+x+y+z$, each two of which have just the point $T^{2}\left(p^{\prime}\right)$ in common. Furthermore

$$
T^{2}\left(p^{\prime} x\right) \cdot T\left(p^{\prime} x\right) \cdot p^{\prime} x=x
$$

$$
T^{2}\left(p^{\prime} y\right) \cdot T\left(p^{\prime} y\right) \cdot p^{\prime} y=y, \quad \text { and } \quad T^{2}\left(p^{\prime} z\right) \cdot T\left(p^{\prime} z\right) \cdot p^{\prime} z=z .
$$

Now it is easy to see that the sum of these nine arcs form the well known graph ${ }^{2}$ ) containing 6 points of order 3 , which is not topologically contained in the plane. Thus the supposition that our. theorem is not true leads to a contradiction.

Theorem 6. If $M$ is a plane continuous eurve and $T(M)=M$ is a homeomorphism and $G=\left[C_{i}\right]_{-\infty}^{+\infty}$ is an infinite rotation group under $T$, then $F\left(C_{i}\right)$ reduces to one point and, for any preassigned positive number $\varepsilon, \delta\left(O_{i}\right)<\varepsilon$ for all save a finite number of subscripts $i$.

1) See G. T. Whyburn, Ooncerning plane closed point sets which are accessible from certain subsets of their complements, Proc. N. A. S. (1927), p. 659, Theorem 3.

2) See C. Kuratowski, Sur le problème des courbes gauches en Topologie, Fund. Math. 15 (1930), pp. 271-291.

Fundamenta Mathematicae, T. Xxvill. 
Proof. In the first place, whether $M$ lies in a plane or not $F\left(G_{i}\right)$ must be connected, since by virtue of Theorem 2 we have

$$
F\left(C_{i}\right)=F\left(\sum C_{j}\right)=\lim C_{j}
$$

and the last set clearly is connected since each $C_{j}$ is connected.

Therefore if $F\left(C_{i}\right)$ contains more than one point, it must contain more than two points and, hence, by Theorem $5, G$ would have to be of order $\leqq 2$. whereas $G$ is infinite. Therefore $F\left(C_{l}\right)=p$ where $p$ is a point of $K$.

The second part of the theorem follows immediately from (1).

Theorem \%. If $M$ is a two dimensional sphere and $T(M)=M$ is a homeomorphism such that one rotation group under $T$. is of order $>1$, then $K$ is a simple closed curve ${ }^{1}$ ).

Proof. Take a rotation group of order $>1$ and call two of its elements $C_{1}$ and $C_{2}$. Then by Theorem $4, F\left(C_{1}\right)$ is contained in a simple closed curve $J$ and, since $C_{1}$ is open in $M$ and therefore $F\left(C_{1}\right)$ separates $M$, it follows that $F\left(C_{1}\right)=J=F\left(C_{2}\right)$. Now we show that $C_{1}+C_{2}+J=M$. By the Jordan Curve Theorem, $M-J=D_{1}+D_{2}$, where $D_{1}$ and $D_{2}$ are connected regions. Now $C_{1}$ is contained either in $D_{1}$ or in $D_{2}$, say in $D_{1}$; then clearly $C_{1}=D_{1}$ since $D_{1}$ is connected and contains no point of $F\left(C_{1}\right)$. Similarly $C_{2}=D_{2}$. Whence $C_{1}+C_{2}+J=M$ and therefore $J=K$.

Corollary. Under conditions of Theorem 7, there exists only one rotation group under $T$ and this group is of order 2 .

1) Since this theorem was obtained the author has learned that a similar conclusion under less general conditions has been proven independently by $W$. Dancer and extended by R. L. Wilder. The same method used by Wilder to extend $D$ ancer's result may be used to extend this result to higher dimensional Euclidean spheres. See abstracts by Dancer and Wilder in the Bulletin of the Amer. Math. Soc., Vol 4l, pp. 342 and 484, respectively and Fund. Math. 27.

University, Va.

\section{Mean values of trigononetrical polynomials.}

By

J. Marcinkiewicz and A. Zygmund (Wilno).

\section{CHAPTER I.}

1. The object of this chapter is to establish a number of inequalities between various mean values of trigonometrical polynomials.

Let $x_{0}<x_{1}<x_{2}<\ldots<x_{2 n}$ be a system of $2 n+1$ points equally distributed $(\bmod 2 \pi)$ over the interval $(0,2 \pi)$, i.e.

$$
x_{\nu}=x_{0}+\nu \frac{2 \pi}{2 n+1} \quad(\nu=0,1, \ldots, 2 n) .
$$

Let

$$
S(x)=\frac{1}{2} a_{0}+\sum_{k=1}^{n}\left(a_{k} \cos k x+b_{k} \sin k x\right)
$$

be an arbitrary trigonometrical polynomial, real or complex, of order not exceeding $n$. It is well known that

$$
\frac{1}{2 \pi} \int_{0}^{2 \pi}|S(x)|^{2} d x=\frac{1}{2 n+1} \sum_{y=0}^{2 n}\left|S\left(x_{p}\right)\right|^{2} .
$$

In this chapter we extend this relation to the case of exponents other than 2. It is plain that the sign of equality in (1) shall have to be replaced by a sign of inequality.

Without loss of generality, we may suppose that $x_{0}=0$, for otherwise it is sufficient to consider the polynomial $S\left(x+x_{0}\right)$ instead of $S(x)$. 